\title{
Three-dimensional magnetic field structure of a flux-emerging region in the solar atmosphere
}

\author{
Rahul Yadav ${ }^{1}$, Jaime de la Cruz Rodríguez ${ }^{1}$, Carlos José Díaz Baso ${ }^{1}$, Avijeet Prasad ${ }^{2}$, Tine Libbrecht ${ }^{1}$, \\ Carolina Robustini ${ }^{1}$, and Andrés Asensio Ramos ${ }^{3}$

\footnotetext{
${ }^{1}$ Institute for Solar Physics, Dept. of Astronomy, Stockholm University, AlbaNova University Centre, 10691 Stockholm, Sweden e-mail: rahul.yadav@astro.su.se

2 Center for Space Plasma and Aeronomic Research, The University of Alabama in Huntsville, Huntsville, Alabama 35899, USA
} \\ 3 Instituto de Astrofisica de Canarias, Via Lactea s/n, 38205 La Laguna, Tenerife, Spain
}

Received 26 September 2019 / Accepted 29 October 2019

\begin{abstract}
We analyze high-resolution spectropolarimetric observations of a flux-emerging region (FER) in order to understand its magnetic and kinematic structure. Our spectropolarimetric observations in the He I $10830 \AA$ Apectral region of a FER were recorded with GRIS at the $1.5 \mathrm{~m}$ aperture GREGOR telescope. A Milne-Eddington-based inversion code was employed to extract the photospheric information of the Si I spectral line, whereas the He I triplet line was analyzed with the Hazel inversion code, which takes into account the joint action of the Hanle and the Zeeman effects. The spectropolarimetric analysis of the Si I line reveals a complex magnetic structure near the vicinity of the FER, where a weak $(350-600 \mathrm{G})$ and horizontal magnetic field was observed. In contrast to the photosphere, the analysis of the He I triplet presents a smooth variation of the magnetic field vector (ranging from 100 to $400 \mathrm{G}$ ) and velocities across the FER. Moreover, we find supersonic downflows of $\sim 40 \mathrm{~km} \mathrm{~s}^{-1}$ appearing near the foot points of loops connecting two pores of opposite polarity, whereas strong upflows of $22 \mathrm{~km} \mathrm{~s}^{-1}$ appear near the apex of the loops. At the location of supersonic downflows in the chromosphere, we observed downflows of $3 \mathrm{~km} \mathrm{~s}^{-1}$ in the photosphere. Furthermore, nonforce-free field extrapolations were performed separately at two layers in order to understand the magnetic field topology of the FER. We determine, using extrapolations from the photosphere and the observed chromospheric magnetic field, that the average formation height of the He I triplet line is $\sim 2 \mathrm{Mm}$ from the solar surface. The reconstructed loops using photospheric extrapolations along an arch filament system have a maximum height of $\sim 10.5 \mathrm{Mm}$ from the solar surface with a foot-point separation of $\sim 19 \mathrm{Mm}$, whereas the loops reconstructed using chromospheric extrapolations reach around $\sim 8.4 \mathrm{Mm}$ above the solar surface with a foot-point separation of $\sim 16 \mathrm{Mm}$ at the chromospheric height. The magnetic topology in the FER suggests the presence of small-scale loops beneath the large loops. Under suitable conditions, due to magnetic reconnection, these loops can trigger various heating events in the vicinity of the FER.
\end{abstract}

Key words. Sun: magnetic fields - Sun: chromosphere - Sun: infrared

\section{Introduction}

The emergence of magnetic flux at the solar surface plays a vital role in understanding the formation of active regions and general solar activity (Zwaan 1985; van Driel-Gesztelyi \& Green 2015). Magnetic flux-emerging regions (FERs) are also important to understand the dynamic coupling between different layers of solar atmosphere as they connect interior and outer solar atmosphere (Cheung \& Isobe 2014). Flux-emerging regions, or in general active regions, are formed by the rise of flux tubes from the convection zone to the solar surface due to magnetic buoyancy or Parker instability (Parker 1955). These flux tubes, when piercing the solar surface, form bipolar regions and generate a serpentine-like configuration over a wide range of spatial scales. On granular scales (1-2 Mm), due to granular convection, many small-scale bipolar or uni-polar magnetic structures emerge or disappear (Bernasconi et al. 2002). On large scales ( 100 Mm) however, the interaction between photospheric motions and the cancellation or coalescence of small bipolar or unipolar regions leads to the formation of active regions (Caligari et al. 1995; Fan 2004).

In the chromosphere, emerged loops in a FER are commonly known as arch filament systems (AFSs). They appear archshaped when observed using the line core filtergrams of $\mathrm{H} \alpha$, Ca II H \& K, and He I $10830 \AA$ A. These emerged loops become dark due to their high density of chromospheric material, which leads to enhanced absorption under favorable conditions. Generally, the length of AFSs or magnetic loops can lie between 20 and $30 \mathrm{Mm}$, whereas their width can reach a few megameters. The maximum height of AFS loops can vary between 5 and $25 \mathrm{Mm}$ with a lifetime of about 30 min (Bruzek 1967). Supersonic downflow (30-50 $\mathrm{km} \mathrm{s}^{-1}$ ) has been observed near both foot-points of an AFS, whereas near the middle part of loops strong upflows $\left(2-20 \mathrm{~km} \mathrm{~s}^{-1}\right.$ ) have been observed by various authors (Bruzek 1967; Solanki et al. 2003; González Manrique et al. 2018). For the structure and dynamics of AFSs, see a review by Chou (1993) and references therein.

Photospheric magnetic field measurements, mainly dominated by the Zeeman effect, are commonly available from various space- and ground-based telescopes. However, similar measurements in the chromosphere and corona are challenging due to low magnetic field strength and the scarcity of sensitive spectral lines (Solanki et al. 2006; Wiegelmann et al. 2014). Fortunately, the He I $10830 \AA$ triplet line offers a unique diagnostic of the upper chromosphere. The excitation of the He I triplet line in the upper chromosphere is partially due to photoionization from EUV radiation and to collisional excitation in regions with electron temperatures higher than 20000 K (Goldberg 1939; Zirin 1975; Andretta \& Jones 1997; 
Centeno et al. 2008; Leenaarts et al. 2016). The magnetically sensitive He I $10830 \AA$ multiplet originates between the lower $2^{3} \mathrm{~S}_{1}$ level and upper $2^{3} \mathrm{P}_{2,1,0}$ level. It consists of a blue component at $10829.0911 \AA$, and two blended red components at $10830.2501 \AA$ and $10830.3397 \AA$. The linear polarization of the He I triplet is dominated by atomic level polarization, and in the presence of a magnetic field this atomic level polarization is modified by the Hanle effect (Trujillo Bueno et al. 2002). Circular polarization in the He I $10830 \AA$ lines is dominated by the longitudinal Zeeman effect, whereas the linear polarization is generally caused by the joint action of the transverse Zeeman effect and atomic level polarization even for the magnetic strength of $\sim 1 \mathrm{kG}$ (Trujillo Bueno \& Asensio Ramos 2007). However, for stronger field strengths the linear polarization of He I triplet is dominated by the transverse Zeeman effect.

The helium triplet line has been used by several different authors to investigate various chromospheric features. For example, magnetic loops (Solanki et al. 2003; Xu et al. 2010), supersonic downflows (Lagg et al. 2007), the chromosphere above sunspots (Schad et al. 2015; Joshi et al. 2016), flares (Kuckein et al. 2015; Judge et al. 2015), spicules (López Ariste \& Casini 2005; Trujillo Bueno et al. 2005; Centeno et al. 2010; Martínez González et al. 2012; Judge et al. 2015; Orozco Suárez et al. 2015), prominences (Bommier et al. 1981; Casini et al. 2003; Merenda et al. 2006; Orozco Suárez et al. 2014; Martínez González et al. 2015), and filaments (Kuckein et al. 2009; Sasso et al. 2014; Díaz Baso et al. 2019). Furthermore, the He I triplet line within the coronal loops can be used for coronal magnetic field measurements (Schad et al. 2016; Schad 2018).

The first inference of a magnetic field vector in an FER in the upper chromosphere was presented by Solanki et al. (2003), derived by inverting the full Stokes profiles of the He I $10830 \AA$ triplet. Assuming that the emerged loops are directed along the magnetic field lines, using the inferred magnetic field vector these latter authors reconstructed full 3D structures of magnetic loops in the solar atmosphere. They reported that the reconstructed loops are $\approx 10 \mathrm{Mm}$ high above the solar surface with strong downflows near the foot-points. Furthermore, the magnetic field strength decreased with height in both legs from 400 and $500 \mathrm{G}$ at the chromospheric foot-points to below $50 \mathrm{G}$ at the apex. Later, Judge (2009) presented a measurement of a magnetic field at a nearly constant height of $\approx 2.4 \mathrm{Mm}$, not along magnetic loops. In order to check the arguments provided by Solanki et al. (2003), another study was performed in a different active region by $\mathrm{Xu}$ et al. (2010). These latter authors also observed that the reconstructed loops are aligned with the long dark features visible in the He I triplet. To further remove the discrepancy over the height at which the He I absorption takes place, Merenda et al. (2011) investigated the influence of the height on the strength of the Stokes $Q$ and $U$ profiles. These authors found that the linear polarization signals are well reproduced if the loop apex is higher than $\sim 5 \mathrm{Mm}$, and concluded that the dark features in FER are tracing the emerged loops.

Although investigations of FERs at the photospheric layers have been performed by various authors, spectropolarimetric analyses in the upper atmosphere are still limited (see Cheung \& Isobe 2014, and references therein). Therefore, in order to enhance our understanding of FERs in the upper solar atmosphere we present the spectropolarimetric analysis of a FER. The main motivation of this article is to understand the kinematic and magnetic structure of a FER in the photosphere and the chromosphere. Moreover, in order to understand the magnetic topology in FERs, the extrapolations are performed separately at two layers using the measured magnetograms. Even though analyses of FERs or active regions using single layer (photosphere) extrapolations have been performed by various authors (Wiegelmann \& Sakurai 2012, and references therein), very few investigations have explored two-layer extrapolations. This analysis of an FER using two-layer extrapolation confers several advantages. It can allow us to check the reliability of the extrapolations, to determine the relative formation height of spectral lines, and to test the consistency between the magnetic field observed at two different layers (Yelles Chaouche et al. 2012).

This article is organized in the following manner. An overview of observations is described in Sect. 2. The analysis of spectropolarimetric data is discussed in Sect. 3. The extrapolations using the observed magnetograms are described in Sect. 4. Finally, the results and a discussion are presented in Sects. 5 and 6, respectively.

\section{Observations}

A FER located near the solar limb, $X=-789^{\prime \prime}$ and $Y=224^{\prime \prime}$ ( $\mu=0.52$ ), was recorded between 10:36 and 10:48 UT on 3 June, 2015. The observations were performed using the GREGOR Infrared Spectrograph (GRIS, Collados et al. 2012) at the $1.5 \mathrm{~m}$ aperture GREGOR telescope at Observatorio del Teide, Tenerife (Schmidt et al. 2012). The GRIS recorded the full Stokes parameters $(I, Q, U$, and $V)$ along the spectrograph slit. The observed spectral range contains two photospheric lines ( $\mathrm{Si}$ I and $\mathrm{Ca}$ I) and the chromospheric He I $10830 \AA$ triplet line. The spectral sampling along the wavelength axis was $18 \mathrm{~m} \AA$ per pixel. The spatial pixel size and step size along the slit was 0 '!135. The spatial scan was performed along the perpendicular direction of the slit in a total of 250 steps, resulting a field of view (FoV) of $34^{\prime \prime} \times 60.5^{\prime \prime}$. The observed FoV is outlined in Fig. 1.

\subsection{Data reduction}

Standard calibration routines were applied to the raw data, which include the correction for dark current, flat-field, polarimetric calibration, wavelength calibration, and correction of residual fringes (Collados 2003). In order to improve the signal-to-noise ratio $(\mathrm{S} / \mathrm{N})$, we averaged $2 \times 2$ spatial pixels and 2 spectral pixels, which results in spatial and spectral resolutions of 0 ".27 and $36 \mathrm{~m} \AA$ per pixel, respectively. After these corrections, we employed the PCA de-noising technique (Martínez González et al. 2008) to further improve the $\mathrm{S} / \mathrm{N}$ in Stokes parameters, which yields a noise level in Stokes $(Q, U$, and $V)$ of the order of $(4,4,6) \times 10^{-4} I_{\mathrm{c}}$, where $I_{\mathrm{c}}$ is the continuum intensity. A relative velocity calibration was performed by fitting an averaged Stokes I profile of the Si line, which was obtained by taking the average of intensity profiles over a quiet-sun region in the scanned FoV.

Although the above procedure reduces the noise in the observed data significantly, we noticed that there were some spurious short-period and small-amplitude fringes still present over the entire spectral range, mainly in the Stokes $I$ profiles. One of the reasons for the appearance of these fringes could be multiple reflections on the surface of the optical devices placed in the optical path. In order to remove the contribution of these fringes in Stokes I profiles we employed the Relevent Vector Machine method (RVM; Tipping 2000). This method, described in detail in Díaz Baso et al. (2019), works in the following manner. The original signal, in our case the intensity spectrum, is decomposed into two components: one without fringes and another 


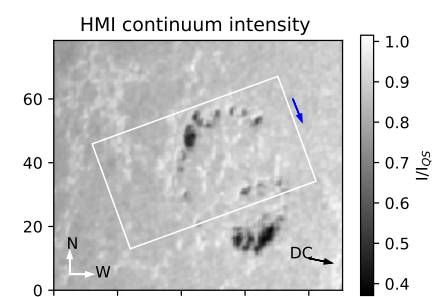

AIA $171 \AA$

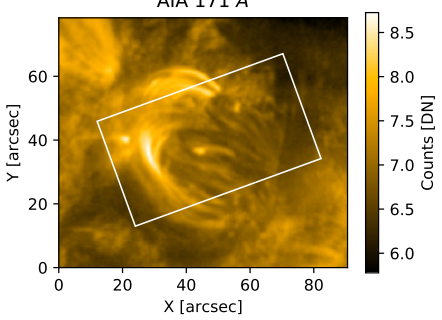

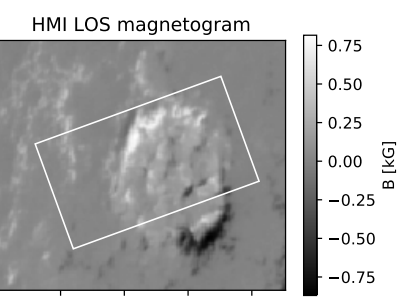

AIA $1700 \AA$

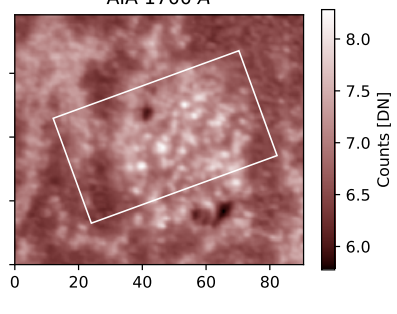

Fig. 1. Overview of the observations at 10:36 UT on 3 June, 2015. Top panels: HMI continuum image and LOS magnetogram of the FER. Bottom panels: AIA $171 \AA$ and $1700 \AA$ Amages of the FER. A white box rectangle outlines the FoV covered by GRIS. Black and blue arrows in the top left panel indicate the direction of the solar disk center and the scanning direction of the slit, respectively. Solar north and west directions are indicated by $\mathrm{N}$ and $\mathrm{W}$.

with only fringes. These two components are represented by two dictionaries (sets of functions) of different properties. The first dictionary is made of Gaussian functions with different widths centered at several wavelength positions across the spectrum. On the other hand, the second dictionary is made of sine and cosine functions of different periods. The observed spectrum is then fitted by taking the coefficients of these two dictionaries as free parameters (see Eq. (4) in Díaz Baso et al. 2019). Lastly, the first dictionary made of Gaussian functions gives the spectrum free from fringes, whereas the second dictionary shows the presence of fringes in the spectrum.

\subsection{Overview of observations}

Figure 1 shows an overview of the observation. The top and bottom panels show contextual images obtained with the Helioseismic and Magnetic Imager (HMI, Scherrer et al. 2012) and the Atmospheric Imaging Assembly (AIA, Lemen et al. 2012), both are onboard the Solar Dynamic Observatory (SDO, Pesnell et al. 2012). A white rectangle superimposed on the images represents the observed FoV. The HMI and AIA maps clearly show the complex structure of the FER. The sequential images obtained from HMI line-of-sight (LOS) magnetogram reveal patches of opposite polarity, moving, merging, and evolving with time, which leads to the enhancement in magnetic field strength and the development of pores. In the AIA EUV $171 \AA$ map, a small-scale loop connecting the emerging bipoles can be clearly identified. During flux emergence, various brightening events are noticed in different SDO/AIA filtergrams (304, 1600 and $1700 \AA$ ). For instance, as shown in Fig. 1, the AIA $1700 \AA$ filtergram reveals several transient brightening events mainly located near the location of mixed polarities.

In order to study the history of the FER, we estimated the magnetic flux rate of the FoV by integrating the positive and negative polarity fluxes separately. We analyzed their temporal evolution a few days before and after the observations performed with GRIS. For this purpose, we adopted the magnetic parameters obtained from the Space-weather HMI Active Region

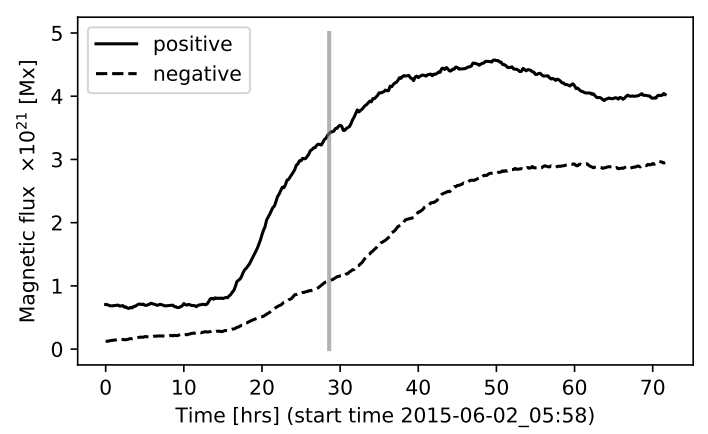

Fig. 2. Temporal evolution of positive and negative fluxes in the FER (solid and dashed lines, respectively). A vertical gray solid line indicates the time of observations with GREGOR.

Patch (SHARP, Bobra et al. 2014), which is derived from fulldisk HMI data. The SHARP images are deprojected to the heliographic coordinates with a Lambert (cylindrical equal area; CEA) projection method. The calculated magnetic flux rate for positive (black solid line) and negative (dashed black line) polarities, using the $B_{z}$ component of the magnetic field strength, are depicted in Fig. 2. We summed only those pixels with $\left|B_{z}\right|$ values larger than $30 \mathrm{G}$, neglecting the contribution of weak signals (Hoeksema et al. 2014). During the flux emergence period, the flux of either polarity has increased up to $\sim 10^{21} \mathrm{Mx}$. Furthermore, for either polarity, the estimated flux emergence rate during the GRIS observation turned out to be $\sim 10^{16} \mathrm{Mx} \mathrm{s}^{-1}$. It is evident from Fig. 2 that the spectropolarimetric observations with GRIS/GREGOR were performed roughly in the middle part of the flux emerging period, which is represented by a solid vertical gray line.

Figure 3 shows the four Stokes maps observed across the He I line. These maps were generated near the core of the red component of the He I triplet. All maps were normalized with the continuum value of the quiet-sun region. The dark elongated features connecting pores of opposite polarity are illustrated in the top panel of Fig. 3. These loops are usually seen above the FER in chromospheric lines because the dense and cool plasma along the loop absorb the radiation coming from below. It is also evident from Fig. 3 that Stokes $Q$ and $V$ have sufficient signal in the loops, and therefore they can be used to infer the magnetic field vector. Our analysis of the spectropolarimetric data is described in the following section.

\section{Analysis of spectropolarimetric data}

We analyzed the spectropolarimetric data of Si I $10827 \AA$ and He I $10830 \AA$ lines in order to infer the physical properties of FERs in the photosphere and chromosphere, respectively.

\subsection{Inversion of the Siı $10827 \AA$ line}

We first inferred the thermodynamic and magnetic parameters of the photosphere using the Si I $10827 \AA$ line. All the Stokes profiles are inverted using the SPIN code (Yadav et al. 2017), which is based on the analytic solution of the radiative transfer equation for polarized radiation in a Milne-Eddington (M-E) model atmosphere (Auer et al. 1977; del Toro Iniesta 2003). The code fits the Stokes profiles by varying the eight free parameters of a model atmosphere: the magnetic field strength $(B)$, its inclination $(\theta)$ and azimuth angle $(\phi)$, line-of-sight (LOS) velocity $\left(V_{\mathrm{LOS}}\right)$, the line-to-continuum absorption ratio $\left(\eta_{0}\right)$, the Doppler width $\left(\Delta \lambda_{\mathrm{D}}\right)$, damping constant $(a)$, source function $\left(S_{0}\right)$ and its 


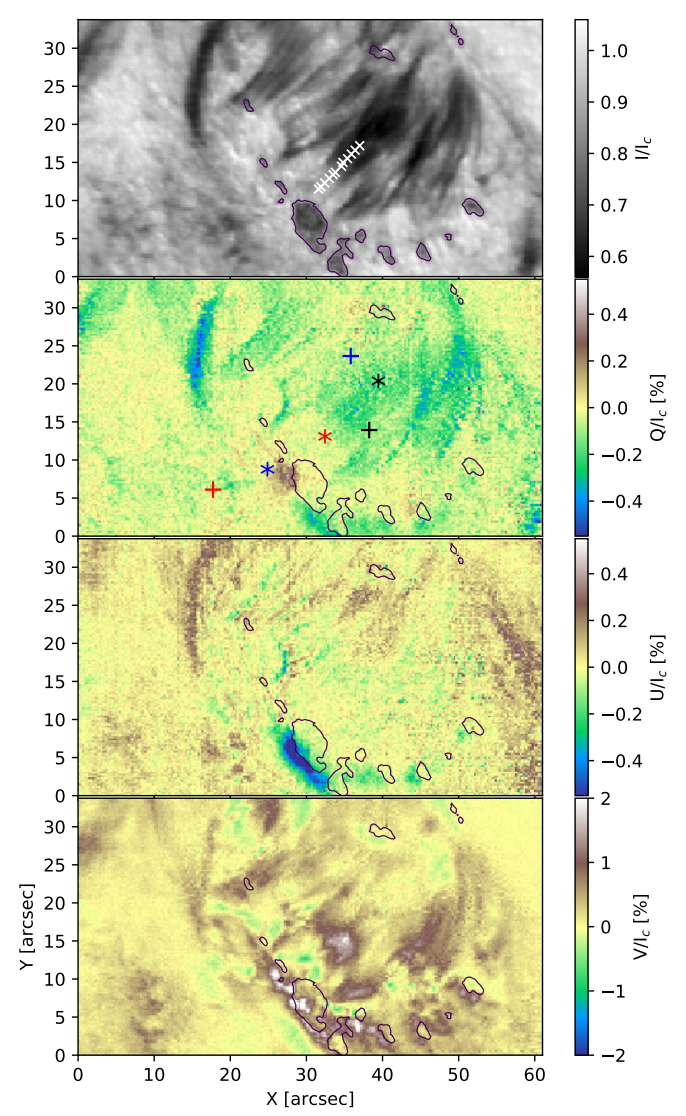

Fig. 3. Stokes $I, Q, U$, and $V$ maps (top to bottom) of the FoV observed with GRIS/GREGOR. Closed contours represent the pores shown in Fig. 1. The three plus symbols and asterisks in one of the panels refer to the inverted pixels shown in Figs. 4 and 5, respectively. In the top panel, white cross symbols indicate the positions of the Stokes I profiles shown in Fig. 6.

gradient $\left(S_{1}\right)$. Before the inversion, all four Stokes profiles were normalized with the averaged quiet-sun continuum intensity. Subsequently, from the Si I line inversion, we retrieve the magnetic field vector and LOS velocity in the photosphere. Generally, the inferred transverse components of magnetic field remain unchanged under a rotation of $180^{\circ}$. This is also known as the $180^{\circ}$ azimuthal ambiguity of the Zeeman effect (Zeeman 1897). Therefore, in order to get the correct orientation of the magnetic field vector, this ambiguity has to be resolved before further investigation. For this purpose, different methods are available; some of them are described in Metcalf et al. (2006). We adopted the automated ambiguity resolution $\operatorname{code}^{1}$ (Leka et al. 2014), which is based on the minimum energy method (Metcalf 1994). After this 180-ambiguity correction, we transformed the magnetic field vector inferred in LOS frame to the solar local reference frame using the transformation matrix given by Gary \& Hagyard (1990).

\subsection{Inversion of the $\mathrm{He} \mathrm{I} 10830 \AA$ line}

The He triplet lines were inverted using the new version of the Hazel inversion $\operatorname{code}^{2}$ (Asensio Ramos et al. 2008). Unlike the previous version, the latest one employed here can synthesize and invert multiple spectral lines simultaneously. It can synthesize photospheric lines under the assumption of local

\footnotetext{
1 Available at https://www. cora.nwra.com/AMBIG/

2 Available at https://github.com/aasensio/hazel2
}

thermodynamic equilibrium, chromospheric lines (e.g., He I triplet) under the multi-term approximation, and a few telluric lines using the Voigt function. The code also uses the emergent intensity from the photosphere, derived using the synthetic module of the Stokes Inversion based on Response functions code (SIR; Ruiz Cobo \& del Toro Iniesta 1992), as an input to the chromospheric model atmosphere. The approach of coupling the photospheric radiation to the chromosphere can generate a more realistic model atmosphere compared to the previous version of the Hazel code.

Even though the stratified model parameters of the photosphere can be retrieved from the Hazel code, we employed a $\mathrm{M}-\mathrm{E}$ based inversion code to invert the Si I line because it provides us with averaged and height-independent model parameters, which are relatively easy to interpret.

To synthesize the Stokes profiles across He I 10830 triplet lines, the Hazel code considers a constant-property slab model of plasma, located at a height " $h$ " above the visible solar surface. It considers that all atoms inside this slab of optical thickness $\tau$ are illuminated from below by the photospheric solar continuum radiation field and the slab is placed in the presence of a deterministic magnetic field strength. The parameters that describe the slab model are the optical depth $(\tau)$ at the core of the red component, the damping parameter $(a)$, the Doppler width $\left(\Delta \lambda_{\mathrm{D}}\right)$, the LOS velocity of the plasma $\left(V_{\mathrm{LOS}}\right)$, the height of the slab from the solar surface, the heliocentric angle, and the three components of the magnetic field vector. Generally, the anisotropic radiation pumping produces population imbalances and quantum coherences between pairs of magnetic sublevels of the He I atoms (Trujillo Bueno \& Asensio Ramos 2007). In addition to this, these levels are influenced by the presence of magnetic field due to the Zeeman and the Hanle effect (Trujillo Bueno et al. 2002). As a consequence of atomic polarization, and both the Zeeman and Hanle effects, the emergent radiation is polarized. To calculate the emergent Stokes profiles $(I=I, Q, U, V)$, the code uses the analytical solution of the radiative equations for a slab of constant properties (Asensio Ramos et al. 2008),

$\boldsymbol{I}(\tau)=\boldsymbol{I}_{0} \cdot \mathrm{e}^{-\boldsymbol{K}^{*} \cdot \tau}+\left[\boldsymbol{K}^{*}\right]^{-1}\left(1-\mathrm{e}^{-\boldsymbol{K}^{*} \cdot \tau}\right) \boldsymbol{S}$,

where $\boldsymbol{I}_{0}$ is the Stokes vector that illuminates the boundary of the slab from below, $\boldsymbol{K}^{*}=\boldsymbol{K} / \eta_{I}$ and $\boldsymbol{S}=\boldsymbol{\epsilon} / \eta_{I} . \boldsymbol{K}$ and $\boldsymbol{S}$ are the propagation matrix and the source function, respectively, and $\boldsymbol{\epsilon}$ and $\eta_{I}$ represent coefficients of the emission vector and the coefficient of the propagation matrix, respectively. For more details regarding the quantum theory of spectral line polarization and the solution of the above equation, see the monograph by Landi Degl'Innocenti \& Landolfi (2004).

The Stokes $I$ profiles in $10830 \AA$ shown in Fig. 4 illustrate that the Si I $10827 \AA$ line blends with the blue component of the He I triplet, whereas the red component blends with a telluric line (10832.3 $\AA$ ). Therefore, in order to achieve a better fitting of profiles and to infer reliable model parameters, along with the He I triplet lines, we also take into account the Si I and the blended telluric line. The following describes the configuration of the Hazel code and the inversion scheme employed to invert the observed Stokes profiles. All pixels are set to invert using three inversion cycles. The Harvard-Smithsonian Reference model Atmosphere (HSRA; Gingerich et al. 1971) model is used as an initial model atmosphere for the $\mathrm{Si}$ I spectral line. For all the inversion cycles we use three nodes in temperature, two nodes in all the components of the magnetic field vector, two nodes in line-of-sight velocity, and one node in microturbulence. For the Si I line we only considered one component of model atmosphere. For the 

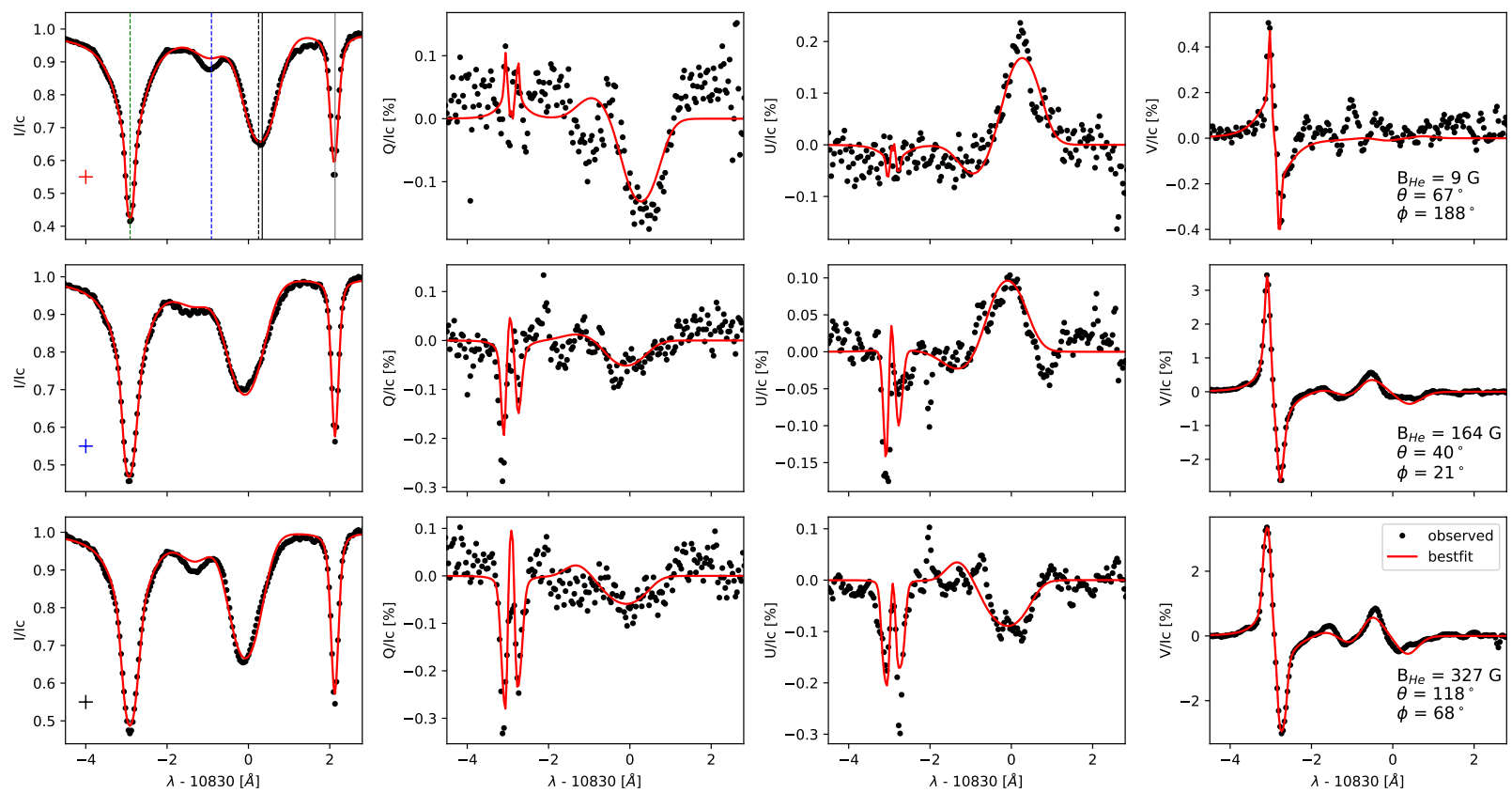

Fig. 4. Observed and best-fit Stokes profiles (dark dotted and red solid lines respectively). All observed profiles were fitted with a single slab of model atmosphere and the inferred magnetic parameters (using He triplet) are given in the right column. The locations of the observed Stokes profiles in each panel are indicated by plus symbols of different colors in Fig. 3. In the top left panel (Stokes I profile), the dashed green and solid gray line represent the Si I $10827 \AA$ and the telluric line (10832 $\mathrm{A}$ ). The rest wavelength of blue (dashed blue) and two blended red components (black dashed and solid) of He I triplet lines are indicated by vertical lines.

He I triplet line however, as a first exercise, all the pixels are inverted using a single-slab model atmosphere placed at a fixed height of 3 " from the solar surface. In addition to this, we assume that the telluric line is well modeled by the Voigt function. The above configuration can invert the whole spectral region around $10830 \AA$ and can be summarized as the light emerging from the photosphere passing to the chromosphere and finally being absorbed by the telluric contamination.

In order to reduce the number of free parameters, we fixed only the damping parameter $(a)$ to $10^{-4}$ value. For the He triplet lines, Lagg et al. (2004) also demonstrated that the inferred parameters are not significantly affected by fixing $a$. In the first cycle, only thermodynamic parameters $\left(\Delta \lambda_{\mathrm{D}}, \tau\right.$, and $\left.V_{L O S}\right)$ are allowed to fit the intensity profile by fixing $B_{x}, B_{y}$, and $B_{z}$. In our case, a single inversion cycle is generally sufficient to retrieve the thermodynamic parameters. In the next two cycles, where the outputs of the previous cycle are considered as the initial model input, we also allow the magnetic parameters to fit the polarized signals. Further, to avoid the possibility of local minimum of the $\chi^{2}$, we performed the inversion several times using different Stokes weights and initial guess models. As an example, Fig. 4 shows the observed Stokes profiles and the best-fit ones, retrieved using the Hazel code. Figures 4 and 5 illustrate how the shape of the Stokes $Q$ and $U$ profiles across the He I triplet lines are generated due to both the Zeeman and the Hanle effect. A single lobe in Stokes $Q$ and $U$ indicates the presence of atomic polarization. The amplitude of this lobe decreases in the presence of magnetic field due to the Hanle effect, which can be seen in Fig. 4. In strong field regions, the triple lobe signature of the Zeeman effect in Stokes $Q$ and $U$ can also be seen in the middle panel of Fig. 5.

Although most of the pixels are well fitted by a single-slab model, we noticed that for some pixels the single-slab model is not suitable to reproduce the observed Stokes profiles of the He I triplet line. The signature of two components is difficult to identify in Stokes $Q, U$, and $V$ due to low signal-to-noise ratio and their complex shapes. Nevertheless, they can be identified in the Stokes I profiles, as shown in Figs. 5 and 6. Generally, the two components have fast and slow velocities. As an example, Fig. 6 demonstrates the appearance of single and double velocity components in the Stokes I profile, one of which (the slow component) is located close to the rest wavelength. We identify these pixels using a threshold value of $\chi^{2}$, obtained after fitting the Stokes I profiles using one and two components of model atmosphere. They are mainly located near the foot-points of loops or near the location of mixed bipolar fields. For these pixels, we take into account the two-slab model atmosphere.

In the Hazel code, two model atmospheres can be combined with the filling factor or as stacked atmospheres. In the latter case, two slab models are placed on top of each other, whereas in the former case they are combined with a filling factor in a single pixel. It has been demonstrated that in a FER various magnetic loops carrying helium plasma can appear at different heights (Solanki et al. 2003; Xu et al. 2010). Under this scenario, the stacking approach can be more realistic. Therefore, in order to fit the two-component profiles we considered the stacking approach instead of the filling factor. We placed the first (slab1) and the second slab (slab2) at heights of $2^{\prime \prime}$ and $3^{\prime \prime}$ from the solar surface, respectively. In this configuration, the light coming from photosphere first passes through slab1, then slab2, and is finally absorbed by the atmosphere of the Earth (see also Eq. (4) in Libbrecht et al. 2017). As an example, the observed and best-fit profiles are shown in Fig. 5. This figure also shows that a single-slab model is unable to fit the observed Stokes profiles; however, using a double-slab model a reasonably good fit can be achieved. For the selected pixels, the inferred parameters using single- and double-slab models are listed in Table 1. The retrieved atmospheric parameters of FER in the photosphere and chromosphere are shown in Fig. 7. After the inversion of the full FoV, the next step is to remove the ambiguity in the retrieved azimuthal angle, which is discussed in the following section. 

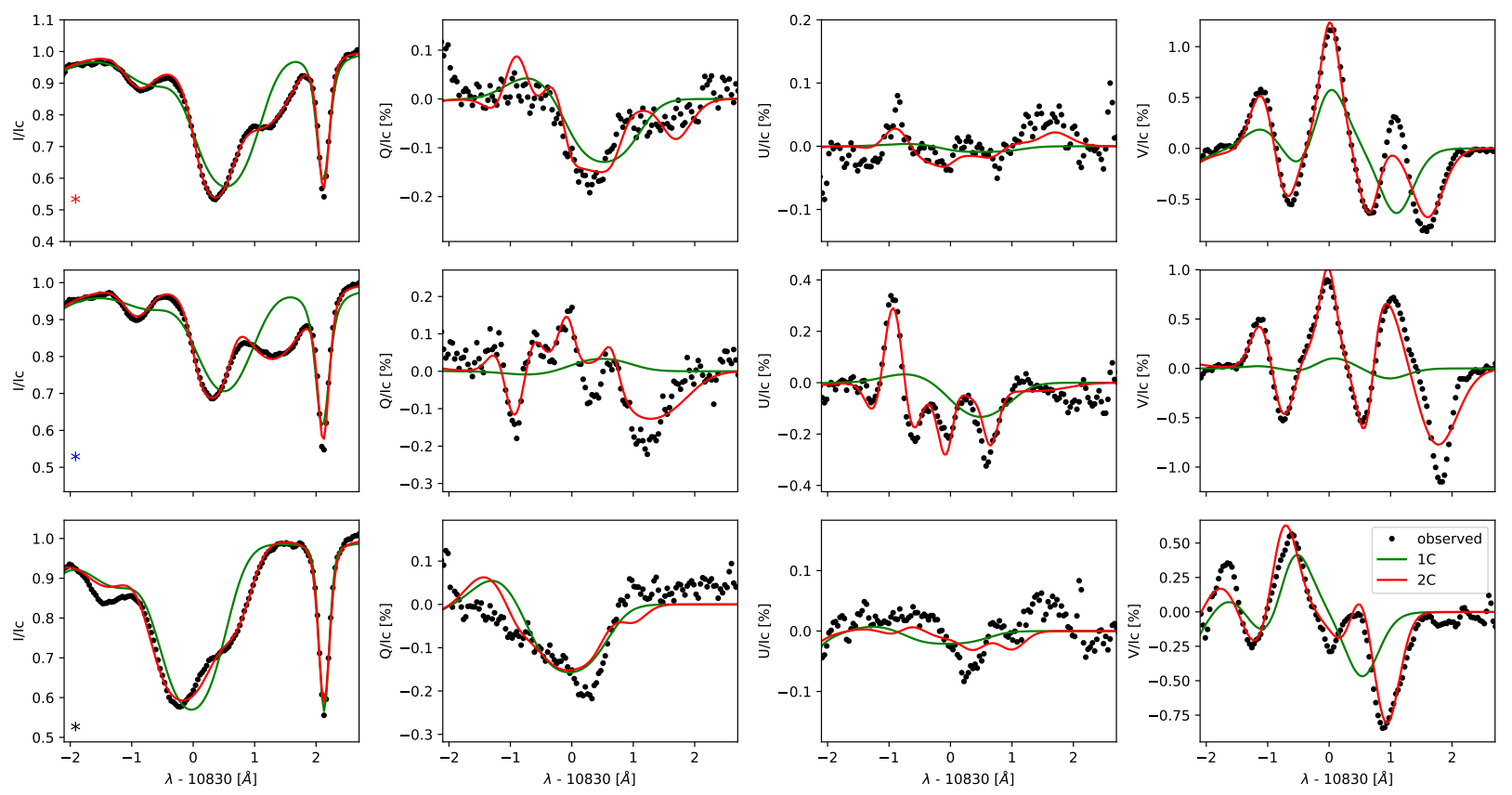

Fig. 5. Same as Fig. 4, but the observed Stokes profiles were fitted using a single slab (solid green line; 1C) and double slab (solid red line; 2C) model atmosphere. The locations of the observed Stokes parameters are indicated by asterisks in Fig. 3 and the retrieved magnetic field vectors and LOS velocities are listed in Table 1.

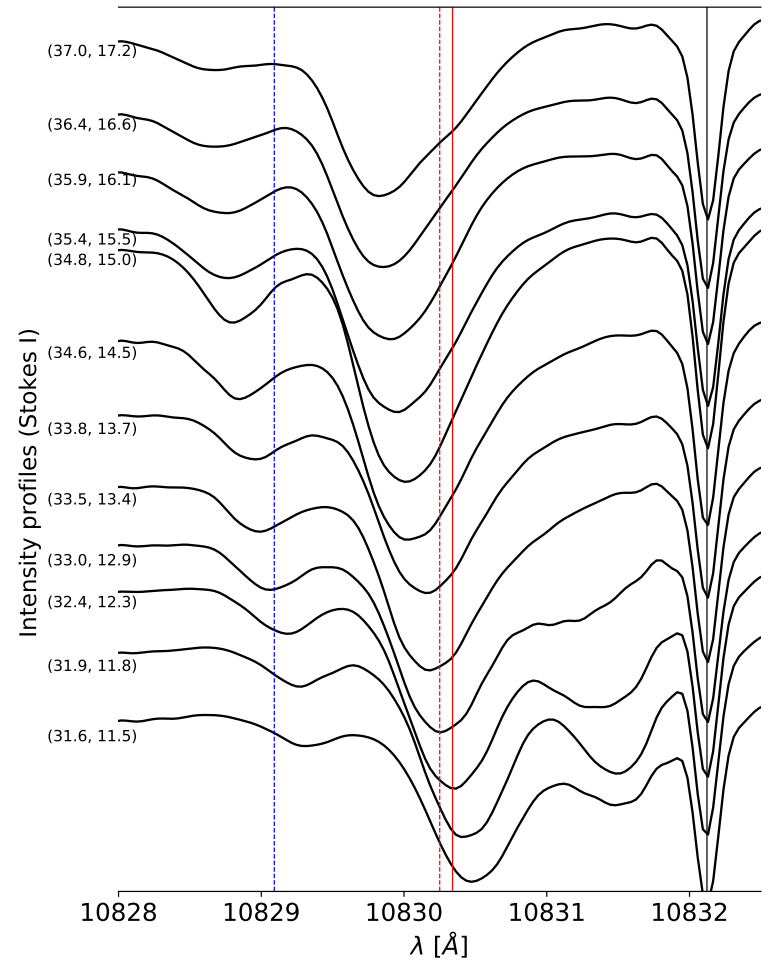

Fig. 6. Stokes $I$ profiles along a loop. At the foot-point (lower profile) two velocity components can be seen. The blue dashed, red dashed, solid red, and solid black lines represent the rest wavelength of the He I triplet lines and the telluric line, respectively. The numbers in parentheses denote the position (in arcsec) of the pixels, which are indicated by white cross symbols in Fig. 3 .

\subsection{Ambiguity resolution}

As mentioned above, at the photospheric height the transverse component of the magnetic field vector is affected by well-known $180^{\circ}$ azimuth ambiguity, which can be resolved using different available methods. In addition to this, the chromospheric azimuthal map inferred from Hazel can have multiple possible solutions, induced by both the Zeeman and the Hanle effects. These solutions depend on the specific regime of the magnetic field and on the scattering geometry of the FoV (Schad et al. 2015; Díaz Baso et al. 2019). The number of solutions can go up to four (or up to eight if the Stokes $V$ signal is under the noise or not given), which can be resolved using different approaches provided by various authors (Solanki et al. 2003; Orozco Suárez et al. 2014; Schad et al. 2015; Martínez González et al. 2015).

To resolve the ambiguity in the He I triplet line we used the azimuth angle of the photospheric extrapolations at a particular height as an initial estimation for our inversion code. This height is estimated by comparing the photospheric extrapolated magnetic field strength (at different heights) with the inferred chromospheric magnetic field. After comparison, we find that the two maps show maximum similarity around $\sim 2 \mathrm{Mm}$ from the solar surface. Using only the direction (azimuth angle, say $\phi_{\text {ex }}$ ) of the extrapolated field lines (at $\sim 2 \mathrm{Mm}$ ) in the initial guess model, we then invert the Stokes profiles again. During inversion, the azimuth angles are allowed to vary in a range $\left(\phi_{\mathrm{ex}} \pm 20^{\circ}\right)$ so that the solution lies close to $\phi_{\mathrm{ex}}$. Subsequently, a visual inspection was performed between the photospheric and chromospheric magnetograms, which reveals good agreement overall (see Figs. 8 and 9). The obtained similarity suggests that the ambiguity resolution for the $\mathrm{He}$ line is reliable. We note that the chromospheric maps shown in Figs. 7 and 8 are obtained using a single-slab model atmosphere. The ambiguity-corrected, inverted maps, both for the photosphere and the chromosphere, are analyzed below.

\section{Nonforce-free extrapolations}

The plasma $\beta$ parameter, which is the ratio of thermal to magnetic pressure, can be of the order of unity in the photosphere (Gary 2001). This indicates that the plasma in the photosphere 
R. Yadav et al.: Three-dimensional magnetic field structure of a flux-emerging region in the solar atmosphere

Table 1. Parameters retrieved by a single-slab and double-slab model atmosphere.

\begin{tabular}{|c|c|c|c|c|c|c|c|c|c|c|c|c|}
\hline & \multicolumn{4}{|c|}{1 component } & \multicolumn{8}{|c|}{2 component } \\
\hline & $B[\mathrm{G}]$ & $\theta\left[{ }^{\circ}\right]$ & $\phi\left[^{\circ}\right]$ & $V_{\mathrm{LOS}}\left[\mathrm{km} \mathrm{s}^{-1}\right]$ & $B[\mathrm{G}]$ & $\theta\left[{ }^{\circ}\right]$ & $\begin{array}{l}\text { Slab1 } \\
\phi\left[^{\circ}\right]\end{array}$ & $V_{\mathrm{LOS}}\left[\mathrm{km} \mathrm{s}^{-1}\right]$ & $B[\mathrm{G}]$ & $\theta\left[{ }^{\circ}\right]$ & $\begin{array}{l}\text { Slab2 } \\
\phi\left[^{\circ}\right]\end{array}$ & $V_{\mathrm{LOS}}\left[\mathrm{km} \mathrm{s}^{-1}\right]$ \\
\hline * & 307 & 68.1 & 94 & 6.2 & 829 & 68.9 & 32.3 & 20 & 402 & 57.7 & 45.9 & -0.9 \\
\hline$*$ & 483 & 53.9 & 19 & 6 & 654 & 44.3 & 48.1 & 27.1 & 760 & 75.1 & 95.6 & -0.1 \\
\hline$*$ & 241 & 117 & 42.4 & -9.1 & 343 & 60 & 42.4 & -12.8 & 515 & 56.7 & 58.3 & 10 \\
\hline
\end{tabular}

Notes. The locations of inverted pixels are indicated by asterisks in Fig. 3, whereas the observed and fitted profiles are shown in Fig. 5.
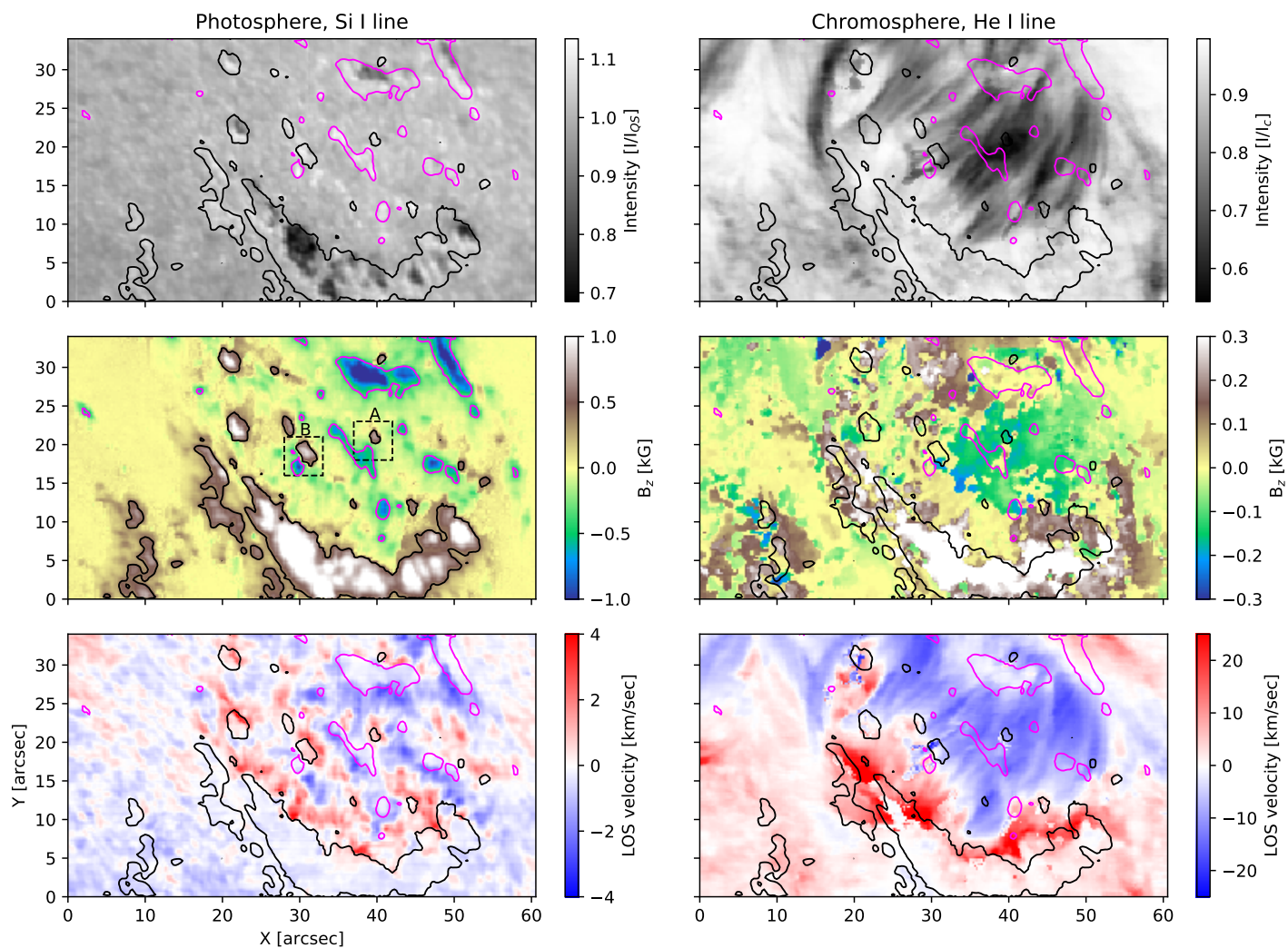

Fig. 7. Left panel: inferred photospheric parameters using M-E inversion of the Si I line. Right panel: inferred parameters obtained by inverting He triplet lines using the Hazel code. The magenta and black closed contours in each map represent the negative and positive polarity of the magnetic field observed in the photosphere, respectively. Square boxes (A and B) indicate the location of mixed-polarity regions.

can be in a nonforce-free state. Thus, in the photosphere, the retrieved current density and magnetic energy in force-free extrapolations would not be reliable (Peter et al. 2015). In order to better understand the magnetic field topology in FER, we modeled the magnetic field using nonforce-free field extrapolations (NFFF; Hu \& Dasgupta 2008; Hu et al. 2010). The NFFF code is based on the principle of minimum dissipation rate (MDR), which is derived from a variational problem (Shaikh et al. 2008). Although the full MDR-based approach requires the measurement of two successive layers of vector magnetograph on the solar surface, the same code can be modified for the single layer as demonstrated by Hu et al. (2010). This code constructs the magnetic field lines as the superposition of two linear force free fields (LFFFs) and one potential field, $\boldsymbol{B}=\boldsymbol{B}_{1}+\boldsymbol{B}_{2}+\boldsymbol{B}_{3}$, where each $\boldsymbol{B}_{i}(i=1,2,3)$ obeys the following equations,

$\nabla \times \boldsymbol{B}=\alpha \boldsymbol{B} ; \quad \nabla \cdot \boldsymbol{B}=0$,

where $\alpha$, the so-called force-free parameter, is a function of space. Without a loss of generality, we can set one of the three sub-fields (say, $\boldsymbol{B}_{1}$ ) to be potential by choosing $\alpha_{1}=0$. While the other non-potential pair satisfies, $\alpha_{2} \neq \alpha_{3} \neq 0$. The optimal pair $\left(\alpha_{2}, \alpha_{3}\right)$ is then obtained by minimizing the following quantity,

$E_{n}=\frac{\sum_{i=1}^{M}\left|\boldsymbol{B}_{t, i}-\boldsymbol{b}_{t, i}\right|}{\sum_{i=1}^{M}\left|\boldsymbol{B}_{t, i}\right|}$,

where $M$ represents the total number of grid points on the transverse plane. Further, $\boldsymbol{b}_{t}$ and $\boldsymbol{B}_{t}$ are the computed and measured transverse magnetic field vectors on the bottom boundary, respectively. Further details of the code and its application to the solar active regions are given in $\mathrm{Hu} \&$ Dasgupta (2008) and $\mathrm{Hu}$ et al. (2010).

In the following we describe the extrapolations of magnetic field lines using the magnetograms observed in the photosphere and the chromosphere. We first employ the photospheric vector magnetogram, retrieved by inverting the Si I 10827 line using M-E approximation, as the lower boundary condition for NFFF extrapolations. Computations are performed in a box of $226 \times$ $125 \times 125$ grid points in the $x, y$, and $z$ directions, respectively. The distance between two grid points in the horizontal 

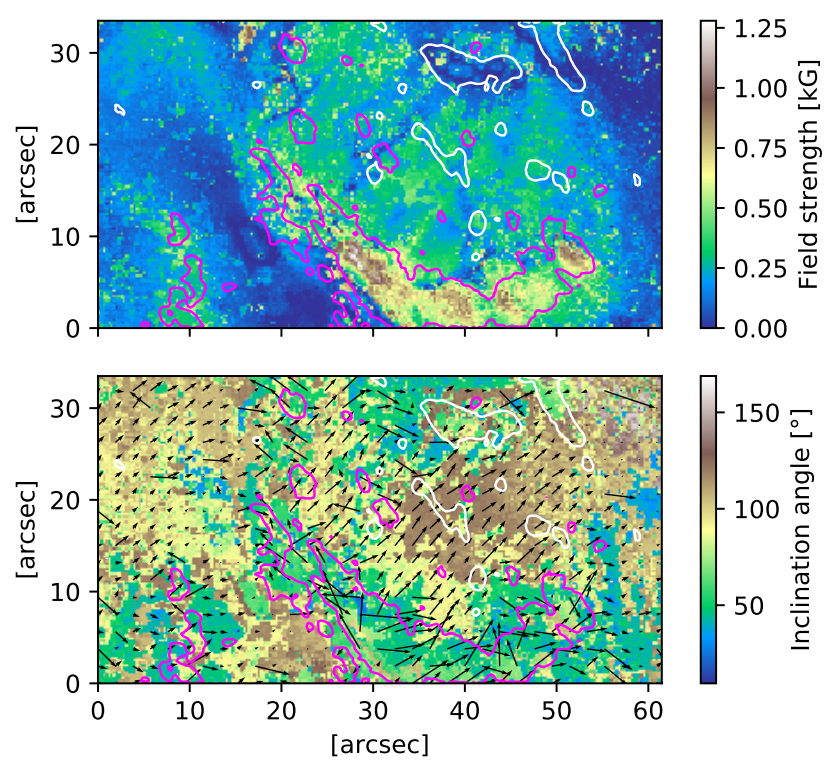

Fig. 8. Top panel: field strength retrieved by inverting the He I triplet line. Bottom panel: inclination angle (in background) and horizontal magnetic field vectors are shown as black arrows. The closed white and magenta color contours represent negative and positive polarities in the FoV at the photospheric level.
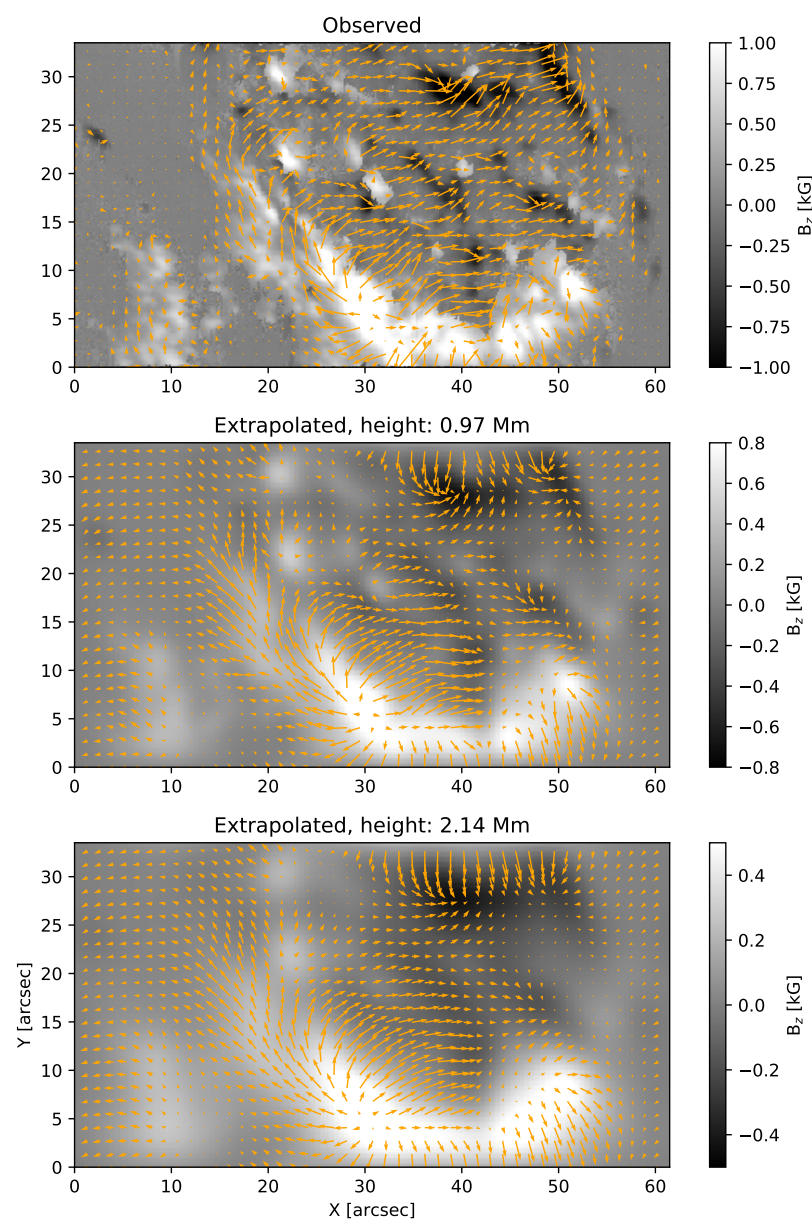

Fig. 9. Top panel: observed $B_{z}$ components of the photospheric magnetic field derived from Milne-Eddington inversion of the Si I line. Middle and bottom panels: $B_{z}$ components of the photospheric magnetic field constructed using the NFFF extrapolation at different heights. The arrows show the orientation of the magnetic field vector, where their length is proportional to the horizontal field strength.

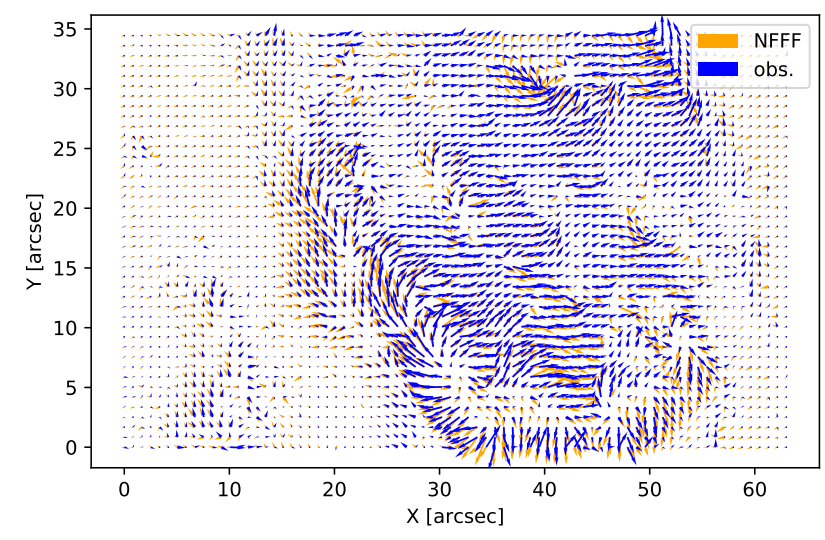

Fig. 10. Comparison between the observed and extrapolated horizontal magnetic field. Blue arrows represent the observed horizontal magnetic field derived using the Si I line, whereas orange arrows represent the horizontal magnetic field obtained from extrapolations at the photospheric boundary $(z=0)$.

and vertical directions is $0 \prime 227(\sim 200 \mathrm{~km})$. For the photospheric extrapolation, the obtained $E_{n}$ value is 0.07 , suggesting that the observed and the extrapolated magnetograms at the photospheric level $(z=0)$ are remarkably similar. As an example of extrapolations starting from the photosphere, Fig. 9 (top panel; in background) shows the observed $B_{z}$ component of the photospheric magnetic field, whereas the middle and bottom panels depict the extrapolated $B_{z}$ components of the constructed magnetic field using the NFFF at different heights. In each panel, the arrows represent the direction of the magnetic field vector, and their length is proportional to the horizontal field strength. This figure also demonstrates that for the higher regions the magnetic field strength decreases and the field vector becomes smoother, which is generally observed in extrapolations. The similarity between the observed and extrapolated horizontal magnetic field is shown in Fig. 10. In this figure, blue arrows represent the observed horizontal magnetic field derived from the inversion of the Si I line, whereas orange arrows represent the horizontal magnetic field obtained from extrapolations at the photospheric boundary $(z=0)$. It is evident from the figure that the observed and computed extrapolations are remarkably similar at the lower boundary $(z=0)$, yielding a linear Pearson correlation coefficient value of 0.97 . This comparison also ensures us that the ambiguity correction is reliable for the $\mathrm{Si} I$ line.

Although various authors have used extrapolations starting from a photospheric magnetogram, only a few studies have explored the magnetic topology using the chromospheric magnetogram as a lower boundary condition. Recently, using the photospheric and the chromospheric magnetograms as a lower boundary condition, two independent non-LFFF extrapolations were performed by Yelles Chaouche et al. (2012) to understand the magnetic field topology of an active region filament. In addition to photospheric extrapolation, we also performed independent extrapolations using a chromospheric vector magnetogram, which is inferred by inverting the helium triplet line as a lower boundary condition. We used the same computational domain as described above for the photospheric extrapolations. The $E_{n}$ value for this extrapolation was 0.56 . The higher value in this case as compared to the photospheric field is expected as the field strength is weaker in the chromosphere and the uncertainties in the transverse field are larger. The observed magnetogram is relatively small and not isolated from the active region. The region is therefore far from the flux-balance condition, which is one 

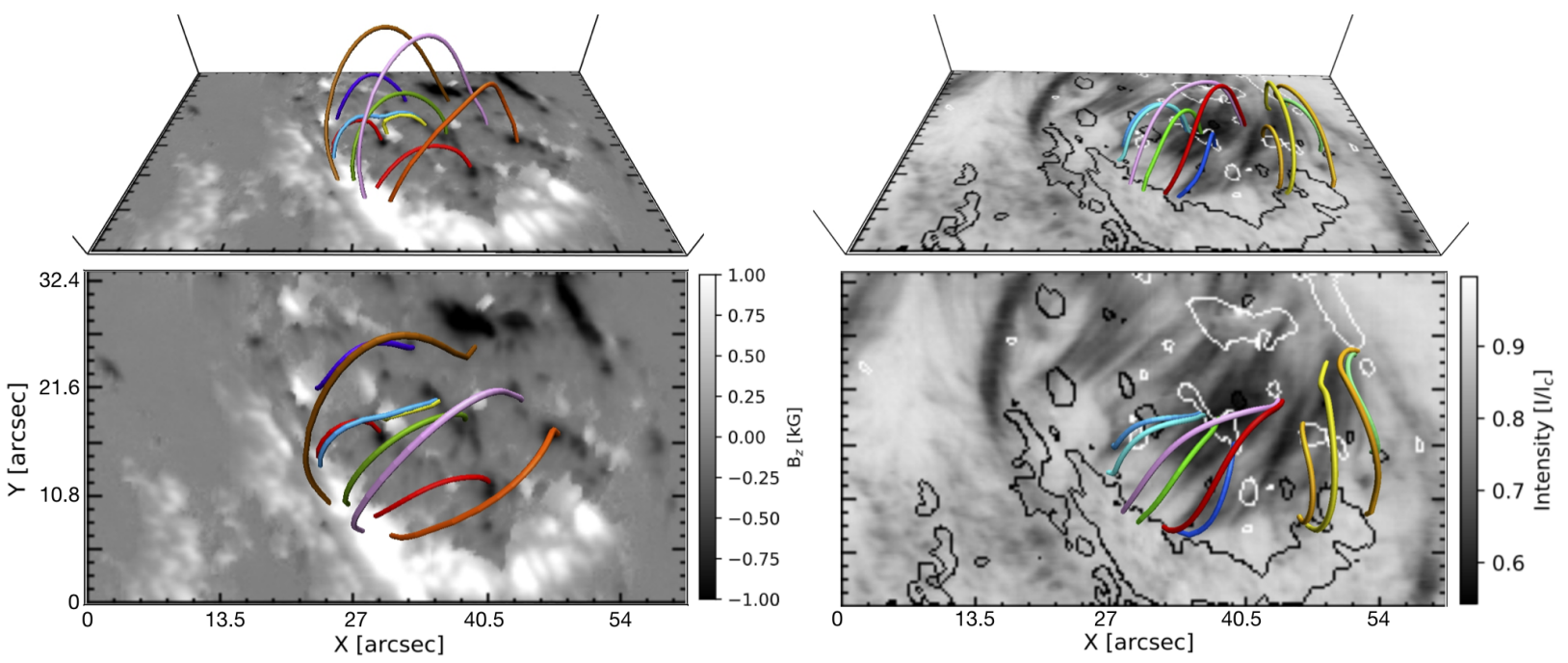

Fig. 11. Three-dimensional view of magnetic field lines. Left panels: extrapolated field lines computed using the observed photospheric magnetic field as a lower boundary condition (top panel: lateral view, bottom panel: top view). The $B_{z}$ component of the magnetic field strength is shown in the background. Right panels: extrapolated field lines computed using the observed chromospheric magnetic field as a lower boundary condition (top panel: lateral view, bottom panel: top view). The intensity image in the background shows He absorption features along the loops; closed black and white contours represent the locations of photospheric positive and negative polarity, respectively.

of the assumptions used in the extrapolations. This means that the extrapolated results near the boundary can lead to inaccurate field line topology. We therefore mainly consider the region a few arcseconds away from the FoV boundaries. The extrapolated field lines computed using the observed chromospheric magnetic field as a lower boundary condition are shown in Fig. 11.

\section{Results}

\subsection{Magnetic field in the photosphere and chromosphere}

The magnetic parameters in the FER, retrieved using the Si I line, exhibit a complex magnetic structure in the photosphere. The $B_{z}$ component of the magnetic field in the photosphere illustrates that there are a few magnetic bipolar features (MBFs) in the FER, two of which are highlighted in boxes A and B (Fig. 7). The polarity of MBFs close to a pore is opposite to the polarity of the pore itself. In contrast to the photospheric structure, the magnetic field in the chromosphere is relatively smooth and exhibits no signature of MBFs. The latter suggests that the features of the MBF are more prominent in the photosphere than in the chromosphere, which is consistent with results from previous studies (Bernasconi et al. 2002; Solanki et al. 2003; Xu et al. 2010) Furthermore, the magnetic field inferred in the photosphere and in the chromosphere displays a weak and horizontal magnetic field in the FER. However, in the photosphere, stronger and more vertical magnetic fields are located in pores $(\sim 1300 \mathrm{G})$ and MBFs $(\sim 800 \mathrm{G})$, whereas in the vicinity of the FER the strength of the magnetic field varies from 350 to $600 \mathrm{G}$. Generally, in the solar atmosphere, the magnetic field strength decreases with height. For instance, the mean field strength value of a pore $(\sim 1350 \mathrm{G})$ observed in the photosphere (located at $X=30^{\prime \prime}, Y=7^{\prime \prime}$ ) drops to $880 \mathrm{G}$ in the chromosphere, whereas the magnetic field strength ranging from 100 to $400 \mathrm{G}$ in the FER decreases by a factor of $\sim 0.3-0.6$ compared to the photosphere. If the average distance between the Si I and He I formation heights is assumed to be 1000-1500 km (Schmidt et al. 1994; Bloomfield et al. 2007; Xu et al. 2010; Joshi et al. $2016)$, then the vertical gradient $(\Delta B / \Delta z)$ in the FER turns out to be $\sim 0.2 \mathrm{G} \mathrm{km}^{-1}$, which is consistent with the values presented by different authors (Xu et al. 2010; Joshi et al. 2016).

\subsection{Line-of-sight velocity in the photosphere and chromosphere}

The inferred LOS velocity in the photosphere and chromosphere is shown in Fig. 7. In the photosphere, between the opposite polarity pores or around the mixed-polarity regions the plasma shows upflows and downflows. These flows demonstrate the expansion of the FER in the photosphere when the pores push through the surrounding plasma. Downflows (up to $3.3 \mathrm{~km} \mathrm{~s}^{-1}$ ) are generally located near the pores of positive polarity, whereas upflows (up to $2.2 \mathrm{~km} \mathrm{~s}^{-1}$ ) are located near the negative pore or negative-polarity region. In contrast to the photosphere, LOS velocities in the upper chromosphere are relatively smooth. Near the foot-points of the elongated dark loops, connecting pores of opposite polarity, we found strong downflows (reaching supersonic velocities of $40 \mathrm{~km} \mathrm{~s}^{-1}$ ), whereas strong upflows ( of $22 \mathrm{~km} \mathrm{~s}^{-1}$ ) are observed near the middle part of the loop.

As an example, the intensity profiles of the He triplet line along a dark featured loop are shown in Fig. 6, where the values in parentheses denote the selected position of the profiles. The top profile represents the loop apex, whereas the bottom profile represents the loop foot-point. The loop apex is strongly blueshifted (upflows). Moreover, this blueshift gradually shifts towards red (downflows) as a function of decreasing loop height. Near the footpoints the profiles are strongly redshifted showing two velocity components. The presence of two components of velocity near the location of loop foot-points was also observed by various authors (Lagg et al. 2007; Xu et al. 2010; González Manrique et al. 2018). The strong upflows near the tops of freshly emerged loops suggest that they carry cool material to the upper atmosphere, which is not yet heated to high temperature. Also, strong downflows near the foot-points suggest that the loop is still rising and chromospheric cool gas is draining through the loop legs, which is consistent with the interpretation from previous studies (Schmidt et al. 2000; Solanki et al. 2003; Xu et al. 2010; González Manrique et al. 2018). 
The overall chromospheric velocity map is remarkably different from the photospheric velocity map. However, in contrast to Solanki et al. (2003), Lagg et al. (2007), and González Manrique et al. (2018), we observed photospheric downflows near the location of strong chromospheric downflows. The maximum photospheric downflows observed at these location were of $3.3 \mathrm{~km} \mathrm{~s}^{-1}$, which is around twice the value reported by $\mathrm{Xu}$ et al. (2010).

\subsection{Magnetic field topology and extrapolated field lines}

In addition to the reconstruction technique described by Solanki et al. (2003), the field lines can also be reconstructed using extrapolation techniques as demonstrated in Wiegelmann et al. (2005). Therefore, in order to understand the magnetic field topology in the FER, we also reconstructed the magnetic field lines using extrapolation approximations. A three-dimensional view of photospheric and chromospheric extrapolated field lines in the FER is shown in Fig. 11. Although open and closed field lines are present in the FoV, for clarity we depict only selected closed field lines. The figure demonstrates that a normal magnetic field configuration is obtained, where the field lines start from a positive-polarity region and stop near a negative-polarity region. Visual inspection reveals that almost all closed extrapolated field lines are parallel to the elongated dark features (see also Leenaarts et al. 2015 for the relation between $\mathrm{H} \alpha$ fibrils and the magnetic field lines in a three-dimensional magnetohydrodynamics simulation of a network region).

Using the photospheric extrapolations we estimated the average formation height of the He line in the FER. We first compared the magnetic field strength in the photospheric extrapolation cube at various heights with the inferred chromospheric one. We found that they show maximum correlation around $\sim 1.5 \mathrm{Mm}$. If we assume that the averaged formation height of the Si I line in a FER is $0.5 \mathrm{Mm}$ from the solar surface $(\tau=1$; Bard \& Carlsson 2008), then the averaged formation height of the He I turns out to be $2 \mathrm{Mm}(1.5 \mathrm{Mm}+$ the formation height of the Si I line) from the solar surface.

Noticeably, many field lines, reconstructed using photospheric and chromospheric extrapolations, start and end around same locations. Under normal field configurations the footpoints of field lines seen in the photosphere and the chromosphere should lie around the same polarity regions, which is supported by our extrapolations. The maximum height of loops reconstructed using photospheric extrapolation is $10.5 \mathrm{Mm}$ above the solar surface with the foot-point separation of $16 \mathrm{Mm}$. Furthermore, as shown in Fig. 11, there are several small loops connecting pores to nearby magnetic structures of opposite polarity. For instance, one of the small loops has a foot-point separation of $5 \mathrm{Mm}$ and is $\sim 2 \mathrm{Mm}$ high.

On the other hand, the magnetic loops reconstructed using the chromospheric extrapolations exhibit similar topology of field lines. These show that the maximum height of a reconstructed loop along an AFS is around $8.4 \mathrm{Mm}$ (or 6.4 Mm from the chromospheric height) with a foot-point separation of $16 \mathrm{Mm}$ at the chromospheric height. In our case the extrapolated loops are comparable to the magnetic loops reconstructed by Solanki et al. (2003). These latter authors reconstructed loops of 10Mm in height with a foot-point separation of $20 \mathrm{Mm}$. In addition to this, using the same approach demonstrated by Solanki et al. (2003), in a different FER, Xu et al. (2010) also reconstructed loops that reached $4-4.5 \mathrm{Mm}$ above the solar surface, which is consistent with the present study. Since we analyzed only a limited FoV of the FER, higher loops may exist in the full FER.

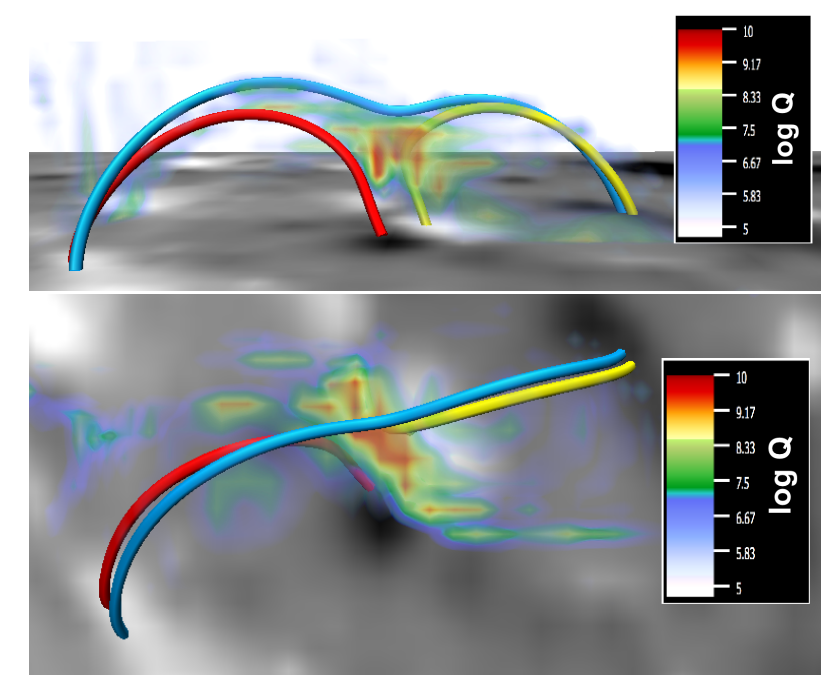

Fig. 12. Top (oblique view) and bottom (top view) panels display the squashing factor around a mixed-polarity region indicated by box B in Fig. 7. White and black background represents the locations of positive and negative polarity in the photosphere, respectively. The closed field lines connecting opposite polarities are shown in different colors.

Further, the loop height heavily depends on strength of $\boldsymbol{B}_{z}$, which may vary from one FER to another.

The photospheric extrapolations reveal that strong electric currents form around mixed-polarity regions. They normally appear at the locations of strong magnetic field gradient. The squashing factor $(Q)$, generally used to quantify this gradient, shows the most probable location of magnetic reconnection. Therefore, we also calculated three-dimensional $Q$ maps using the method of Liu et al. (2016). As an example, we show the top and oblique views of a $Q$ map at the location of a mixed-polarity region (box B in Fig. 7) in Fig. 12, which were generated using the photospheric extrapolations. We observe that intense brightenings in AIA/SDO 1600 and $1700 \AA$ maps are associated with large $Q$ values, which could be Ellerman Bombs (EBs) or UV bursts (Pariat et al. 2004; Vissers et al. 2015; Young et al. 2018). Unfortunately, we did not have sufficient information from other spectral lines to confirm these features as EBs or UV bursts. It is evident from Fig. 12 that large $Q$ values are located on the photospheric inversion line, also known as bald patches (Titov et al. 1993), and they lie in a region 1-1.6 Mm above the solar surface. On the other hand, using chromospheric extrapolations we did not observe strong currents and signatures of magnetic reconnection in the chromosphere. The analysis of the magnetic topology and $Q$ maps suggests that the observed brightenings are generated through magnetic reconnection in the lower chromosphere or upper photosphere, which is consistent with previous suggestions (Georgoulis et al. 2002; Pariat et al. 2004; Vissers et al. 2013, 2019; de la Cruz Rodríguez et al. 2015; Toriumi et al. 2017; Tian et al. 2018).

\section{Discussion and conclusions}

In this study, we analyzed spectropolarimetric observations of a young FER located close to the solar limb. We analyzed the magnetic and kinematic nature of a FER in the photosphere and chromosphere using the Si I $10827 \AA$ and He I $10830 \AA$ triplet lines. In order to retrieve the physical properties of solar atmosphere, the $\mathrm{Si}$ I line is inverted using Milne-Eddington atmosphere, 


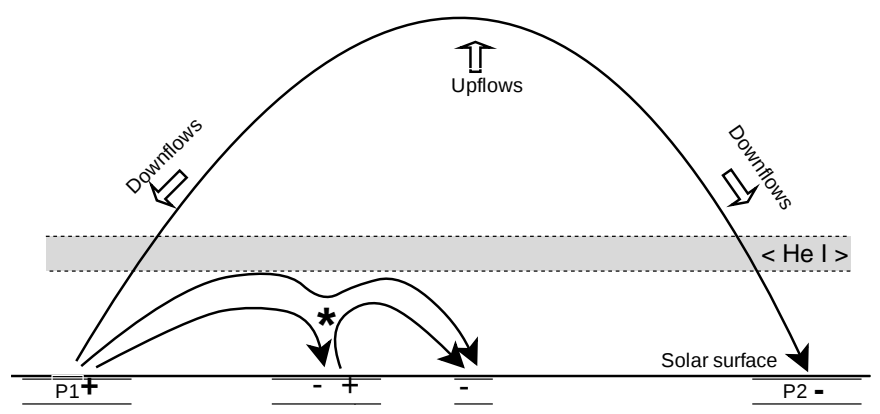

Fig. 13. Sketch of the magnetic field configuration in a FER based on the two-layer extrapolations. The "+" and "-" symbols represent positive and negative polarity on the solar surface, respectively. The gray area outlined by dashed lines represents the average formation height of the He I layer. An AFS connecting two pores (P1 and P2) of opposite polarity is represented by a black closed loop. Arrows indicate the upflows and downflows of plasma along an AFS as observed in the $\mathrm{He}$ triplet line. The asterisk indicates the plausible location of magnetic reconnection near a mixed-polarity region.

whereas He I triplet line is inverted using the Hazel inversion code by considering a joint action of the Hanle effect and the Zeeman effect.

Through spectropolarimetric analysis of the SiI line we observed a complex magnetic structure showing a few magnetic bipolar elements near the vicinity of the FER. In the photosphere, the magnetic field is weak $(350-600 \mathrm{G})$ and horizontal around the middle part of the FER. However, it becomes stronger $(\sim 1000 \mathrm{G})$ and more vertical near the pores. The obtained overall features at the photospheric level are consistent with previous results (Solanki et al. 2003; Lagg et al. 2004; Xu et al. 2010). Our analysis of the He I triplet line shows a smooth variation of the magnetic field vector (ranging from 100 to $400 \mathrm{G}$ ) and velocity across the FER. Furthermore, we find supersonic downflows of $\sim 40 \mathrm{~km} \mathrm{~s}^{-1}$ near the foot-points of loops connecting two pores of opposite polarity, while a strong upflow of $\sim 22 \mathrm{~km} \mathrm{~s}^{-1}$ is seen near the middle part of the loops. Recently, González Manrique et al. (2018) studied the evolution of an AFS in a FER using 64 min of observations. Using He I 10830 intensity profiles, these latter authors measured strong upflows (near the central part) and downflows (near the foot-points) in loops. During $30 \mathrm{~min}$ of lifetime, the upflows decrease gradually whereas the downflows increase in the middle stage and again decrease at the later stage. Finally, the AFS vanishes when downflows drop to zero. Based on their observations, the strong upflows in the present study indicate that the loops are young and carry cool material to the upper atmosphere, which is also evident from the strong He I triplet line absorption along the emerged loops.

In contrast to Lagg et al. (2007) and González Manrique et al. (2018), at the location of the supersonic downflows in the chromosphere, we observed downflows of $\sim 3 \mathrm{~km} \mathrm{~s}^{-1}$ in the photosphere. In addition to this, $\mathrm{Xu}$ et al. (2010) also reported photospheric downflows of $1.5 \mathrm{~km} \mathrm{~s}^{-1}$ below regions with chromospheric downflows. The observed strong photospheric downflows could be a consequence of atmospheric disturbances caused by the supersonic downflowing material along the loop foot-points. Moreover, in contrast to Lagg et al. (2007), no signs of emission in the He I line were observed in our analysis, which indicates that the transition of downflowing material from supersonic to sonic velocities lies below the He I line formation.

To understand the magnetic field topology in the FER, we employed NFFF extrapolations starting from our photospheric and chromospheric magnetic field measurements. A normal magnetic configuration (connecting positive- to negativepolarity regions) is observed above the FER. We observed that there are several small- and large-scale magnetic loops, where small-scale loops lie beneath the large-scale loops forming magnetic canopies of various sizes. The magnetic loops reconstructed using photospheric extrapolations along an AFS have a maximum height of $10.5 \mathrm{Mm}$ and a foot-point separation of $19 \mathrm{Mm}$, whereas the loops reconstructed using chromospheric extrapolations are around $8.4 \mathrm{Mm}$ high and have a foot-point separation of $16 \mathrm{Mm}$. These loops are almost aligned along the He I line absorption features, which is consistent with the previous findings. However, in the lower chromosphere, the observations in Ca II $8542 \AA$ revealed that the magnetic vectors are mostly directed along the dark features or fibrils, but not always (de la Cruz Rodríguez \& Socas-Navarro 2011; Asensio Ramos et al. 2017), which could be an effect of the Ca II $8542 \AA$ line forming deeper in the atmosphere.

Small-scale heating events (EBs or UV bursts) and energy releases at different heights have been observed above the FER. Our analysis suggests that the appearance of these events, or the heating of upper layers in the solar atmosphere in general, is likely due to the magnetic reconnection between the magnetic loops of opposite direction. We also observed that the intense small-scale bright features in AIA 1600 and $1700 \AA$ are associated with large $Q$ values, indicating that they are energized by small-scale magnetic reconnections, which is consistent with the previous suggestions. In our analysis, the magnetic field strength is horizontal in the vicinity of the FER, but no significant brightening is observed in the upper chromosphere using the He I triplet line. However, in a recent analysis of FERs using multi-line observations at high spatial and temporal resolution, Leenaarts et al. (2018) reported that the heating rate in the low chromosphere correlates with the strength of the horizontal magnetic field. The role of the magnetic field vector in chromospheric heating is still unclear, and therefore further investigation of FERs or active regions using multi-line observations is required.

Based on the analysis of spectropolarimetric data from two layers, the magnetic field configuration of a FER is sketched in Fig. 13. This cartoon also illustrates the rearrangement of magnetic field lines during reconnection near a mixed-polarity region, which lie in the lower chromosphere or upper photosphere. The overall magnetic topology in the FER shown here is in agreement with previous findings (Solanki et al. 2003; Xu et al. 2010; Toriumi et al. 2017; González Manrique et al. 2018) and supports the theoretical AFS models. In the future we will investigate the magnetic configuration of a FER and the presence of current sheets around the loops using two boundary conditions (at different layers) in a single extrapolation. Moreover, multi-line observations at high-spatial and temporal resolution are required to understand the observational consequences of FER in different layers. The advent of new-generation solar telescopes such as the Daniel K. Inouye Solar Telescope (DKIST; Tritschler et al. 2015) and the European Solar Telescope (EST; Matthews et al. 2016) will be crucial in this regard.

Acknowledgements. We would like to thank the anonymous referee for the comments and suggestions. The $1.5 \mathrm{~m}$ GREGOR solar telescope was built by a German consortium under the leadership of the Kiepenheuer Institut für Sonnenphysik in Freiburg with the Leibniz Institut für Astrophysik Potsdam, the Institut für Astrophysik Göttingen, and the Max-Planck Institut für Sonnensystemforschung in Göttingen as partners, and with contributions by the Instituto de Astrofísica de Canarias and the Astronomical Institute of the Academy of Sciences of the Czech Republic. The observing time at GREGOR was provided by the Trans-National Access and Service Program of the SOLARNET 
project, funded by the European Commission's 7th Framework Program under grant agreement No. 312495. JdlCR is supported by grants from the Swedish Research Council (2015-03994), the Swedish National Space Board (128/15) and the Swedish Civil Contingencies Agency (MSB). This project has received funding from the European Research Council (ERC) under the European Union's Horizon 2020 research and innovation program (SUNMAG, grant agreemen 759548). The Institute for Solar Physics is supported by a grant for research infrastructures of national importance from the Swedish Research Council (registration number 2017-00625). AP acknowledges partial support of NASA grant $80 \mathrm{NSSC} 17 \mathrm{~K} 0016$ and NSF award AGS-1650854. AAR acknowledges financial support from the Spanish Ministerio de Ciencia, Innovación y Universidades through project PGC2018-102108-B-I00 and FEDER funds. We acknowledge the use of the visualization software VAPOR (www.vapor.ucar.edu) for generating relevant graphics. Data and images are courtesy of NASA/SDO and the HMI and AIA science teams. This research has made use of NASA' Astrophysics Data System. We acknowledge the community effort devoted to the development of the following open-source packages that were used in this work: numpy (numpy.org), matplotlib (matplotlib.org) and sunpy (sunpy.org).

\section{References}

Andretta, V., \& Jones, H. P. 1997, ApJ, 489, 375

Asensio Ramos, A., Trujillo Bueno, J., \& Land i Degl'Innocenti, E. 2008, ApJ, 683,542

Asensio Ramos, A., de la Cruz Rodríguez, J., Martínez González, M. J., \& SocasNavarro, H. 2017, A\&A, 599, A133

Auer, L. H., Heasley, J. N., \& House, L. L. 1977, Sol. Phys., 55, 47

Bard, S., \& Carlsson, M. 2008, ApJ, 682, 1376

Bernasconi, P. N., Rust, D. M., Georgoulis, M. K., \& Labonte, B. J. 2002, Sol. Phys., 209, 119

Bloomfield, D. S., Lagg, A., \& Solanki, S. K. 2007, ApJ, 671, 1005

Bobra, M. G., Sun, X., Hoeksema, J. T., et al. 2014, Sol. Phys., 289, 3549

Bommier, V., Sahal-Brechot, S., \& Leroy, J. L. 1981, A\&A, 100, 231

Bruzek, A. 1967, Sol. Phys., 2, 451

Caligari, P., Moreno-Insertis, F., \& Schussler, M. 1995, ApJ, 441, 886

Casini, R., López Ariste, A., Tomczyk, S., \& Lites, B. W. 2003, ApJ, 598, L67

Centeno, R., Trujillo Bueno, J., Uitenbroek, H., \& Collados, M. 2008, ApJ, 677, 742

Centeno, R., Trujillo Bueno, J., \& Asensio Ramos, A. 2010, ApJ, 708, 1579

Cheung, M. C. M., \& Isobe, H. 2014, Liv. Rev. Sol. Phys., 11, 3

Chou, D. Y. 1993, in IAU Colloq. 141: The Magnetic and Velocity Fields of Solar Active Regions, eds. H. Zirin, G. Ai, \& H. Wang, ASP Conf. Ser., 46, 471

Collados, M. V. 2003, in SPIE Conference Series, ed. S. Fineschi, Proc. SPIE, 4843, 55

Collados, M., López, R., Páez, E., et al. 2012, Astron. Nachr., 333, 872

de la Cruz Rodríguez, J., \& Socas-Navarro, H. 2011, A\&A, 527, L8

de la Cruz Rodríguez, J., Hansteen, V., Bellot-Rubio, L., \& Ortiz, A. 2015, ApJ, 810,145

del Toro Iniesta, J. C. 2003, Introduction to Spectropolarimetry (Cambridge: Cambridge University Press), 244

Díaz Baso, C. J., Martínez González, M. J., \& Asensio Ramos, A. 2019, A\&A, 625, A 128

Fan, Y. 2004, Liv. Rev. Sol. Phys., 1,

Gary, G. A. 2001, Sol. Phys., 203, 71

Gary, G. A., \& Hagyard, M. J. 1990, Sol. Phys., 126, 21

Georgoulis, M. K., Rust, D. M., Bernasconi, P. N., \& Schmieder, B. 2002, ApJ, 575,506

Gingerich, O., Noyes, R. W., Kalkofen, W., \& Cuny, Y. 1971, Sol. Phys., 18, 347 Goldberg, L. 1939, ApJ, 89, 673

González Manrique, S. J., Kuckein, C., Collados, M., et al. 2018, A\&A, 617, A55

Hoeksema, J. T., Liu, Y., Hayashi, K., et al. 2014, Sol. Phys., 289, 3483

Hu, Q., \& Dasgupta, B. 2008, Sol. Phys., 247, 87

Hu, Q., Dasgupta, B., Derosa, M. L., Büchner, J., \& Gary, G. A. 2010, J. Atmos. Sol. Terrest. Phys., 72, 219

Joshi, J., Lagg, A., Solanki, S. K., et al. 2016, A\&A, 596, A8

Judge, P. G. 2009, A\&A, 493, 1121

Judge, P. G., Kleint, L., \& Sainz Dalda, A. 2015, ApJ, 814, 100

Kuckein, C., Centeno, R., Martínez Pillet, V., et al. 2009, A\&A, 501, 1113

Kuckein, C., Collados, M., \& Manso Sainz, R. 2015, ApJ, 799, L25

Lagg, A., Woch, J., Krupp, N., \& Solanki, S. K. 2004, A\&A, 414, 1109

Lagg, A., Woch, J., Solanki, S. K., \& Krupp, N. 2007, A\&A, 462, 1147

Landi Degl'Innocenti, E., \& Landolfi, M. 2004, in Polarization in Spectral Lines, Astrophys. Space Sci. Lib., 307
Leenaarts, J., Carlsson, M., \& Rouppe van der Voort, L. 2015, ApJ, 802, 136 Leenaarts, J., Golding, T., Carlsson, M., Libbrecht, T., \& Joshi, J. 2016, A\&A, 594, A104

Leenaarts, J., de la Cruz Rodríguez, J., Danilovic, S., Scharmer, G., \& Carlsson, M. 2018, A\&A, 612, A28

Leka, K. D., Barnes, G., \& Crouch, A. 2014, Astrophysics Source Code Library [record ascl:1404.007]

Lemen, J. R., Title, A. M., Akin, D. J., et al. 2012, Sol. Phys., 275, 17

Libbrecht, T., Joshi, J., Rodríguez, J. D. 1. C., Leenaarts, J., \& Ramos, A. A 2017, A\&A, 598, A33

Liu, R., Kliem, B., Titov, V. S., et al. 2016, ApJ, 818, 148

López Ariste, A., \& Casini, R. 2005, A\&A, 436, 325

Martínez González, M. J., Asensio Ramos, A., Carroll, T. A., et al. 2008, A\&A, 486, 637

Martínez González, M. J., Asensio Ramos, A., Manso Sainz, R., Beck, C., \& Belluzzi, L. 2012, ApJ, 759, 16

Martínez González, M. J., Sainz, R. M., Ramos, A. A., et al. 2015, ApJ, 802, 3

Matthews, S. A., Collados, M., Mathioudakis, M., \& Erdelyi, R. 2016, Proc SPIE, 9908, 990809

Merenda, L., Trujillo Bueno, J., Landi Degl'Innocenti, E., \& Collados, M. 2006, ApJ, 642, 554

Merenda, L., Lagg, A., \& Solanki, S. K. 2011, A\&A, 532, A63

Metcalf, T. R. 1994, Sol. Phys., 155, 235

Metcalf, T. R., Leka, K. D., Barnes, G., et al. 2006, Sol. Phys., 237, 267

Orozco Suárez, D., Asensio Ramos, A., \& Trujillo Bueno, J. 2014, A\&A, 566, A46

Orozco Suárez, D., Asensio Ramos, A., \& Trujillo Bueno, J. 2015, ApJ, 803, L18

Pariat, E., Aulanier, G., Schmieder, B., et al. 2004, ApJ, 614, 1099

Parker, E. N. 1955, ApJ, 121, 491

Pesnell, W. D., Thompson, B. J., \& Chamberlin, P. C. 2012, Sol. Phys., 275, 3

Peter, H., Warnecke, J., Chitta, L. P., \& Cameron, R. H. 2015, A\&A, 584, A68

Ruiz Cobo, B., \& del Toro Iniesta, J. C. 1992, ApJ, 398, 375

Sasso, C., Lagg, A., \& Solanki, S. K. 2014, A\&A, 561, A98

Schad, T. A. 2018, ApJ, 865, 31

Schad, T. A., Penn, M. J., Lin, H., \& Tritschler, A. 2015, Sol. Phys., 290, 1607

Schad, T. A., Penn, M. J., Lin, H., \& Judge, P. G. 2016, ApJ, 833, 5

Scherrer, P. H., Schou, J., Bush, R. I., et al. 2012, Sol. Phys., 275, 207

Schmidt, W., Knoelker, M., \& Westendorp Plaza, C. 1994, A\&A, 287, 229

Schmidt, W., Muglach, K., \& Knölker, M. 2000, ApJ, 544, 567

Schmidt, W., von der Lühe, O., Volkmer, R., et al. 2012, Astron. Nachr., 333, 796

Shaikh, D., Dasgupta, B., Zank, G. P., \& Hu, Q. 2008, Phys. Plasmas, 15, 012306

Solanki, S. K., Lagg, A., Woch, J., Krupp, N., \& Collados, M. 2003, Nature, 425, 692

Solanki, S. K., Inhester, B., \& Schüssler, M. 2006, Rep. Progr. Phys., 69, 563

Tian, H., Zhu, X., Peter, H., et al. 2018, ApJ, 854, 174

Tipping, M. E. 2000, The Relevance Vector Machine (Microsoft Research: Cambridge, UK)

Titov, V. S., Priest, E. R., \& Demoulin, P. 1993, A\&A, 276, 564

Toriumi, S., Katsukawa, Y., \& Cheung, M. C. M. 2017, ApJ, 836, 63

Tritschler, A., Rimmele, T. R., Berukoff, S., et al. 2015, 18th Cambridge Workshop on Cool Stars, Stellar Systems, and the Sun, 18, 933

Trujillo Bueno, J., \& Asensio Ramos, A. 2007, ApJ, 655, 642

Trujillo Bueno, J., Landi Degl'Innocenti, E., Collados, M., Merenda, L., \& Manso Sainz, R. 2002, Nature, 415, 403

Trujillo Bueno, J., Merenda, L., Centeno, R., Collados, M., \& Landi Degl'Innocenti, E. 2005, ApJ, 619, L191

van Driel-Gesztelyi, L., \& Green, L. M. 2015, Liv. Rev. Sol. Phys., 12, 1

Vissers, G. J. M., Rouppe van der Voort, L. H. M., \& Rutten, R. J. 2013, ApJ, 774,32

Vissers, G. J. M., Rouppe van der Voort, L. H. M., Rutten, R. J., Carlsson, M., \& De Pontieu, B. 2015, ApJ, 812, 11

Vissers, G. J. M., de la Cruz Rodríguez, J., Libbrecht, T., et al. 2019, A\&A, 627, A101

Wiegelmann, T., \& Sakurai, T. 2012, Liv. Rev. Sol. Phys., 9, 5

Wiegelmann, T., Lagg, A., Solanki, S. K., Inhester, B., \& Woch, J. 2005, A\&A, 433, 701

Wiegelmann, T., Thalmann, J. K., \& Solanki, S. K. 2014, A\&ARv, 22, 78

Xu, Z., Lagg, A., \& Solanki, S. K. 2010, A\&A, 520, A77

Yadav, R., Mathew, S. K., \& Tiwary, A. R. 2017, Sol. Phys., 292, 105

Yelles Chaouche, L., Kuckein, C., Martínez Pillet, V., \& Moreno-Insertis, F. 2012, ApJ, 748, 23

Young, P. R., Tian, H., Peter, H., et al. 2018, Space Sci. Rev., 214, 120

Zeeman, P. 1897, ApJ, 5, 332

Zirin, H. 1975, ApJ, 199, L63

Zwaan, C. 1985, Sol. Phys., 100, 397 